International Journal of Environment, Agriculture and Biotechnology
Vol-6, Issue-6; Nov-Dec, 2021
J Journal Home Page Available: https://ijeab.com/
Journal DOI: $10.22161 /$ ijeab

Peer Reviewed

\title{
Development and validation of a bovine parainfluenza virus type 3 indirect ELISA
}

\author{
Silvina Soledad Maidana ${ }^{1,2,3, X} *$, Maria Mercedes Odeon ${ }^{1,4, Y}$, Carola Maria Ferrecio ${ }^{1,2}$, \\ Noelia Magali Grazziotto ${ }^{5}$, Eddie Pisano ${ }^{6}$, Irene Alvarez ${ }^{5}$, Lucia Rocha ${ }^{5}$, Gladys \\ Viviana Parreño ${ }^{1,2,5}$, Sonia Alejandra Romera ${ }^{1,2,3,7}$
}

${ }^{1}$ CONICET, Rivadavia 1917 (C1033AAJ), Ciudad Autónoma de Buenos Aires, Argentina. ${ }^{2}$ IVIT (INTA - CONICET), N. Repetto y Los Reseros S/N, CC25 (B1712WAA), Castelar, Buenos Aires, Argentina. ${ }^{3}$ Cátedra de Inmunogenética, Facultad de Ciencias exactas, Químicas y Naturales, Universidad de Morón, Cabildo 134 (B1708JPD) Morón, Buenos Aires, Argentina. ${ }^{4}$ IFAB (INTA - CONICET) EEA Bariloche, Modesta Victoria 4450 (8400), Bariloche, Río Negro, Argentina. ${ }^{5}$ Instituto de Virología, CICV y A - INTA, N. Repetto y Los Reseros S/N, CC25 (B1712WAA), Castelar, Buenos Aires, Argentina. ${ }^{6}$ Laboratorio Regional Bolivar, Pedro Vignau, San Carlos de Bolivar (6550) - Buenos Aires ${ }^{7}$ Cátedra de Inmunología, Universidad del Salvador, Champagnat 1599-Ruta Panamericana-Km 54.5 PilarB1630AHU-Provincia de Buenos Aires, Argentina.

$\mathrm{X}$ and $\mathrm{Y}$ should be considered joint first author

Corresponding author: María Mercedes Odeon; odeon.maria@inta.gob.ar

Received: 15 Oct 2021; Received in revised form: 28 Nov 2021; Accepted: 05 Dec 2021; Available online: 13 Dec 2021 (C)2021 The Author(s). Published by Infogain Publication. This is an open access article under the CC BY license (https://creativecommons.org/licenses/by/4.0/).

\begin{abstract}
Serological assays, including enzyme-linked immunosorbent assays (ELISA), provide an useful tool for screening animals for the presence of antibodies (Abs) against a wide range of infectious agents (including viruses that cause respiratory disease in cattle) and are mainly used in veterinary medicine to assist to the control and disease's monitoring. The aim of the present study was developing and validating one indirect enzyme-linked immunosorbent assay (ELISA) based on semi purified bovine parainfluenza virus type 3 (BPIV3).This test would allow to detect and quantify Abs against PI3 in serum sample from cattle and guinea pigs on both purposes diagnostic and typify/specify the quality of vaccines. The diagnostic sensitivity and specificity from the assay was $88 \%$ and $100 \%$ for bovine samples, using a threshold of corrected optical density, ODc $=0.300$, and $91 \%$ and $100 \%$ for guinea pig samples with a $O D c=0.250$.The intermediate precision expressed as the assays positive control coefficient of variation (CV) was 20\% for bovines and $8.5 \%$ for guinea pigs. Both techniques reproducibility obtained in interlaboratory assays was CV=17\% for bovines and $15 \%$ for guinea pigs, which found the requirements of OIE $(C V<30 \%)$. The efficacy of biological medicinal products, such as vaccines, relies an optimal model testing quality control. The validated ELISAs represents an important tool for testing vaccine quality, and quantifying and controling BPIV3 infections on cattle.
\end{abstract}

Keywords-Bovine, Guinea pig, Indirect ELISA, Parainfluenza Virus Type 3.

\section{INTRODUCTION}

Bovine parainfluenza virus type 3 (BPIV-3) is a Respirovirus, member of Paramyxoviridae family, antigenically related on human parainfluenza virus type 3 (hPIV-3)1. BPIV3 is an endemic infection of cattle, worldwide. Its presence is mostly documented in cattle, although there are investigations in other mammals, as a result of interspecies jumps, including rhinos, sheep, goats, guinea pigs and even humans 12. In Argentina, serological studies showed up a high incidence of antibodies against BPIV3 in cattle from the main livestock breeding regions ${ }^{14,16,22}$.

In addition, positive serology was reported in domestic and wild South American camelids ${ }^{16,3,17,21}$ and 
the virus was isolated from cattle, water buffaloes ${ }^{15}$ and sheep ${ }^{6}$. BPIV3 infections are often asymptomatic, causing respiratory tissue damage and immunosuppression, predisposing animals to severe bacterial pneumonia. In some instances when animals are subjected to high stressful conditions, infection with BPIV3 can contribute to tissue damage, resulting in severe bronchopneumonia from secondary bacterial infections $^{5,8}$. The resulting disease is part of the bovine respiratory disease complex (BRDC) and is considered as the most significant illness associated with feedlot cattle in the USA and possibly worldwide ${ }^{24}$. There is a demand for a fast detection and serological diagnosis of BPIV3 to monitor the presence of the virus and its antibodies in cattle, which is critical in designing suitable interventions and control.

Currently, the hemagglutination inhibition (HAI) is the gold standard technique to evaluate the immune status on a herd against BPIV3; however, this technique is laborious and has low sensitivity. Serological assays, including enzyme-linked immunosorbent assays (ELISA), provide a useful tool to screen animals to find antibodies's (Abs) against a wide range of infectious agents (including viruses that cause respiratory disease in cattle) and are highly used in veterinary medicine to assist on detecting disease in cattle and also in laboratory animal models, such as the guinea pig model validated in Argentina for vaccine potency testing ${ }^{4,18,19}$.

The assay constitutes a critical method for final validation and transference to animal health authorities of the guinea pig model used to evaluate the skill to produce an effective level of immunization in the target species. This involves an alternative method for vaccine potency testing, which is in alignment with the $3 \mathrm{R}$ initiative of refining, reducing and replacing animal experimentation 11

The aim of the present study was the development and statistical validation from an indirect enzyme linked immunosorbent assays (ELISAs) in order to diagnostic and typify / specify the quality of vaccines.

\section{MATERIALS AND METHODS}

\subsection{Experimental design}

A total of 85 bovine and 95 guinea pigs sera with known history have been used throughout every tests run in this work. The validation has included: diagnostic sensitivity and specificity; repeatability and intermediate precision within a laboratory along several years and the reproducibility in interlaboratory assays. To estimate the relevance of the assays, a concordance analysis between the results obtained by ELISA and by hemagglutination inhibition test was conducted.

\subsection{Serum samples}

A total of 85 bovine serum samples (53 positive and 32 negative for antibodies to BPIV3) and 95 guinea pig serum samples (49 positive and 46 negative for antibodies to BPIV3) were run to determine the cut-off of the assay and its associated sensitivity and specificity of the diagnostic. The serologically positive samples belong to naturally infected bovines and experimental hyperimunized guinea pigs. The negative serum samples were obtained from colostrum deprived calves -that were seronegative to BPIV3 for HAI in bovines. Guinea pig negative serum samples were from non-vaccinated or placebo animals.

The protocol was approved by the Institutional Ethical Committee-INTA (CICUAE-INTA N ${ }^{\circ} 33 / 2012$ ).

2.3 Internal bovine sample used as control sera

As there are no bovine reference controls for BPIV3 in order to have positive and negative controls, HAI-titrated sera positive and negative sera were chosen to make pools that were, titrated, aliquoted and stored at $-20^{\circ} \mathrm{C}$. Positive and negative control samples were included in each plate of each ELISA run, the positive control titer (HAI) was 5120 HAIU for bovine pool serum and 10240 HAIU for guinea pig serum pool. The assay was valid if the $\mathrm{Ab}$ titer of the positive control was the expected value \pm standard deviation (2SD) and the negative control was below the cut off of the assay.

\subsection{Hemagglutination inhibition assay}

HAI assays were performed by incubating serial 2-fold dilutions of bovine or guinea pig serum at $25{ }^{\circ} \mathrm{C}$ for 30 min with 8 HA units per $25 \mathrm{ul}$ of BPIV-3. Subsequently, guinea pig red blood cells were added to each well, incubation was continued for $90 \mathrm{~min}$ and each well was then observed for hemagglutination. The sera were treated with kaolin to adsorb nonspecific hemagglutination inhibitors that were found there. It will be taken as the end-point of the serum activity (anti PI-3 HAI neutralizing $\mathrm{Ab}$ titer) the reverse of the maximum dilution in wichhaemagglutination phenomenon was inhibited. HAI titres were expressed as the reciprocal of the highest dilution of serum that was inhibited virus-mediated agglutination of erythrocytes. The final result is expressed in Hemagglutination Inhibitory Units (HAIU). The reciprocal of serum activity end-point dilution, multiplied by the virus hemagglutinating units ( 8 HAIU) will give as a result the number of serum haemagglutination inhibiting units per unit volume. The presence of HAI reactions at dilutions less than 1/10 (40-80 HAIU) are considered negative. Values greater than 1/80 (640 HAIU or more) 
represent infection response or to vaccination (Res. SENASA 598/12).

\subsection{Enzyme linked immunosorbent assay}

MDBK cells were infected with semipurified BPIV3 virus strain SF-4 in laminar flow at an m.o.i. of 0.1 TCID50 per cell and were incubated for 48 hours $37^{\circ} \mathrm{C}$ with $5 \% \mathrm{CO} 2$ in MEM-E, Gibco. After that time, the cytopathic effect of the culture was observed by a light microscope. Cells were lysed with a freeze / thaw process of $-80^{\circ} \mathrm{C}$ for 10 minutes at $37^{\circ} \mathrm{C}$ for three cycles. The culture medium was clarified by low speed centrifugation and supernatant centrifuged at $13216 \mathrm{x} \mathrm{g}$ for $1 \mathrm{~h}$. The pellet was resuspended in $\mathrm{pH}=7 \mathrm{NET}$ buffer (Tris- $\mathrm{HCl} 500 \mathrm{mM}$, Tris Base $\mathrm{NaCl} 100 \mathrm{mM}, \mathrm{NaCl}$ EDTA $10 \mathrm{mM}$ ) and titrated. ELISA plates (Inmulon IB; Dynatech, Laboratories) were coated, in alternating columns with positive antigen (BPIV3 amplified in MDBK cells) and negative antigen (mock: not infected cells). The incubation time was for 18 hours at $4{ }^{\circ} \mathrm{C}$. The blocking solution was about $50 \mathrm{ul}$ of the phosphate buffer solution ( $\mathrm{pH} 7.4)$ supplemented with $0.05 \%$ Tween20 (PBS-T) and ovalbumin 1\% p/v (PBS-T OVA $1 \%$ ) for thirty minutes at $37^{\circ} \mathrm{C}$. The sera sample (1/40) were incubated for an hour. The following step was to add an anti-bovine conjugated with peroxidase (HRP, horseradish peroxidase - Kirkegaard\& Perry Laboratories, KPL) in a $(1: 2000)$ dilution. Once the plate has been washed, it was been developed by adding distilled water, hydrogen peroxide ( $3 \% \mathrm{w} / \mathrm{v} \mathrm{H} 2 \mathrm{O} 2)$ and the orthophenyldiamine(OPD), $0.06 \mathrm{mg} / \mathrm{ml}$ as substrate/chromogen system (Sigma), leaving it to act for about 5 minutes (developing solution) and it was made stopped using $7 \%$ sulfuric acid (H2SO4). The optical density was readspectrophotometrically at $490 \mathrm{~nm}$.

The ELISA assay was adapted for detecting guinea pig antibodies by using the same procedure described on bovine samples. Peroxidase-labeled affinity purified goat anti guinea pig $\operatorname{IgG}(\mathrm{H}+\mathrm{L})$ (Kirkegaard\&PerryLaboratories,KPL) has been used as a conjugate in a 1:4000 working dilution.

\subsection{Statistical methods for ELISA validation}

2.6.1Feasibility studies, initial repeatability and intermediate precision

The repeatability was expressed as the optical density (OD) coefficient variation for positive control sera obtained in the different runs. The control sera were assayed at a 1/40 dilution duplicated on every ELISA run. The assay was valid if the Ab titer of the positive control was the expected value \pm 2 standard deviation (2SD) and the negative control was below the cut off of the assay.

For full validation, the assay's intermediate precision was estimated by bovine and guinea pig positive control sera data, collected from ELISA runs conducted for 3 year in the our lab. For estimating the repeatability and intermediate precision from the ELISA on each species, an ANOVA for a nested model of variance has been carried out. The applied model allowed to quantify the relative contribution of different variation sources (intra assay: same plate and different plates; inter assay: different runs; and different samples: bovine or guinea pig serum) as the relative coefficient of variation $(\mathrm{CV})$.

2.6.2Diagnostic sensitivity and specificity of the ELISA

The assay cut off of and its associated DSe and DSp were estimated basing the frequency (negative and positive sera) distribution of the ELISA values, obtained after analyzing the reference populations of 1/40 dilution. ELISA results were normalized by expressing the raw corrected optical density values (ODc; sample OD-mock $\mathrm{OD}) \mathrm{OD}$ as the percentage of positivity (PP\%) of the highpositive bovine and guinea pig reference control sera described in section "Internal bovine sample used as control sera". The control sera were assigned a $100 \%$ PP\% value and there were included in duplicate in each plate. An ELISA run was taken as valid if the positive control ODc fell on the established admissible working range (mean ODc $\pm 2 \mathrm{SD}$ ). In this study, the assay's sensitivity has been determinated like the probability of positive test results, when the animal was effectively positive for antibodies to BPIV3 (either due to infection or vaccination). Whereas the specificity has been defined like the probability of a negative test result when the animal was effectively negative for antibodies to BPIV3 (Greiner and Gardner, 2000) ${ }^{7}$.

\subsubsection{ROC analysis}

The assay's cut-off selection for both species was also carried out with the aid of the receiver-operating (ROC) analysis, using the MedCalc Statistical Software version 18.11.6 (MedCalc Software bvba, Ostend, Belgium).

\subsubsection{Detection limit and linearity of the assay}

The relative analytical sensitivity or detection limit was defined as the least amount of antibody to BPIV3 detectable in an ELISA run positive sample with already known Ab titer (positive serum). The ELISA Ab titerwas estimated by the end-point, limiting dilution analysis from those reference sera. Briefly, dose response curves were constructed by using a total of 36 replicates of positive bovine serum and 16 replicates positive guinea pig serum, assayed in four-fold dilutions in several plates within different independent ELISA runs. To find out the analytic sensitivity linear regression curves has been determined by plotting the ELISA values of those sera expressed as ODc from positive control versus the logtransformed dilutions.

To verify the linearity of the system, the Mallows'sCpwere used to assess the fit of a regression 
model that has been estimated by using ordinary least squares.

2.6.5 Correlation between the antibody titer determined by ELISA and HAI

The correlation between the BPIV3 antibody titer determined by the HAI and ELISA have been tested in vaccinated animals. The vaccines tested included 2 experimental combined vaccines containing and 107.3 TCID50/dose of inactivated BPIV3 and one commercial vaccine (Biopoligen HS, Biogénesis-Bagó) applied to prevent bovine respiratory diseases. On this study, 90 bovine and 43 guinea pig serum samples were tested.

The relationship between the Ab titer obtained by ELISA and HAI in bovines and guinea pigs were studied. Specifically we have evaluated the Pearson's correlation coefficient between HAI and ELISA Ab titer to BPIV3.

\subsubsection{Reproducibility: inter-laboratory assay}

The inter-laboratory assay included data collected in 3 laboratories for bovine and guinea pigs samples. The laboratories involved were Respiratory Virus and Animal Welfare division, Adventitious virus section, and INCUINTA from the Institute of Virology, INTA, Castelar. The laboratories received the kit and coded samples that included positive and negative controls and standard sera. The laboratories were requested to perform five ELISA assays following the supplied protocol, including 6 plates per run and testing the samples in 3 replicates per plate. The reproducibility of the assay was calculated on the coefficient of variation (relative standard deviation) using a nested ANOVA.

\section{RESULTS}

Table 1. Validation parameters

\begin{tabular}{|c|c|c|c|}
\hline \multirow[t]{2}{*}{ Validation parameters } & \multicolumn{2}{|l|}{ Result } & \multirow[t]{2}{*}{ Acceptance criteria } \\
\hline & Bovine & Guinea Pig & \\
\hline Cut-off (ODc) & 0,3 & 0,25 & \\
\hline Diagnostic specificity & $88 \%$ & $91 \%$ & \\
\hline Diagnostic sensitivity & $100 \%$ & $100 \%$ & \\
\hline Positive control mean ODc & 0,7 & 0,699 & \\
\hline Standard deviation (SD) & 0,08 & 0,09 & \\
\hline $\mathrm{N}$ & 85 & 95 & \\
\hline $\begin{array}{l}\text { ODc admissible working range of the positive } \\
\text { control ( } \bar{y} \pm 1 \text { SD) }\end{array}$ & $0,62-0,78$ & $0,61-0,79$ & \\
\hline Repeatability & $15,00 \%$ & $5,60 \%$ & $C V<20 \%$ \\
\hline Intermediate precision & $20,70 \%$ & $8,56 \%$ & $C V<25 \%$ \\
\hline Linearity & Yes, $R^{2}=0,9, p<0,0001$ & Yes, $R^{2}=0,85, p<0,0001$ & \\
\hline Inter-laboratory assay & (3 labs) & (3 labs) & \\
\hline Reproducibility & $17 \%$ & $15 \%$ & $C V<30 \%$ \\
\hline
\end{tabular}

3.1 Cut-off, diagnostic sensitivity and specificity of the ELISA for bovine and guinea pigs: agreement with HAI

All bovine samples $(n=85)$ were tested with HAI and ELISA. A total of 53 samples were positive for hemagglutination inhibition, while 32 were negative. For the ELISA, 43 out of 53 positives samples gave as a result higher ODc than 0.3 at the 1:40 dilution, and all of negative samples showed up values that were lower than 0.3 . The frequency of positivity distribution percent obtained for positive and negative reference samples, run at 1:40 dilution is depicted in Fig.1a. Using a 0.3 ODc cutoff, the assay showed up a $88 \%$ diagnostic sensitivity and $100 \%$ diagnostic specificity and a high agreement with the HAI assay (kappa: 0.764) (Fig.1b); so it was considered suitable to use as a screening method for field surveys of infected animals. This cut-off was confirmed by ROC analysis conducted by MedCalc $\AA$ software as the threshold that gives the highest accuracy (Table 1 and Fig. 1b). The area under the ROC curve value $(\mathrm{AUC}=0.929, \mathrm{p}=0.0001$ ) indicated that the test has the ability to distinguish between the positive and negative samples with $95 \%$ confidence in the $93 \%$ of the times that a random simple is tested.

For guinea pig samples, the frequency analysis of positivity percent value distribution from reference sera, showed that a 0.25 ODc cut-off gave the highest levels of diagnostic sensitivity and specificity (Fig.1a). This value was also confirmed by ROC analysis (Table 1 and Fig.1b). Similarly to the ROC curve (AUC $=0.995, p=0.0001$ ) obtained for bovine samples, the ELISA has the ability to score properly a guinea pig sample with $95 \%$ confidence in the $97 \%$ of the times that a random simple is tested. Using a 0.25 ODc cut-off the ELISA showed an almost 
perfect agreement with the HAI assay (kappa: 0.811) and a relative sensitivity and specificity of $91 \%$ and $100 \%$, respectively. a

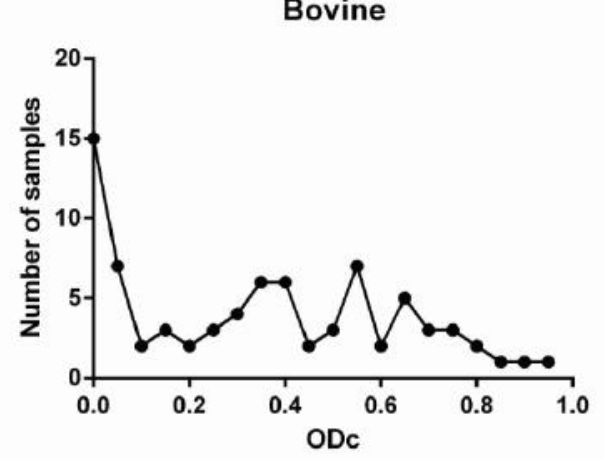

\begin{tabular}{|c|c|c|c|}
\hline \multirow{2}{*}{$\begin{array}{c}\text { ELISA Cut-off } \\
\text { DOC } 0,3\end{array}$} & \multicolumn{3}{|c|}{ Bovine Reference population } \\
\cline { 2 - 4 } & + & - & Total \\
\hline+ & 43 & 0 & 43 \\
\hline- & 10 & 32 & 42 \\
\hline Total & 53 & 32 & 85 \\
\hline Kappa & Dse= $\quad 88 \%$ & DSp= $100 \%$ & \\
\hline \multicolumn{4}{|r}{} \\
\hline
\end{tabular}

b

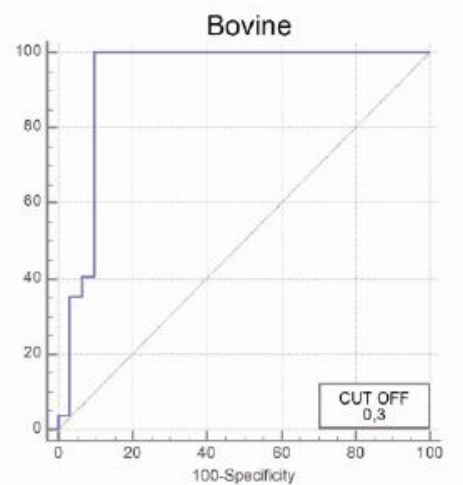

\begin{tabular}{|l|r|}
\hline Area under the ROC curve (AUC) & 0,929 \\
\hline Standard Error a & 0,041 \\
\hline $95 \%$ Confidence interval b & 0,852 to 0,973 \\
\hline z statistic & 10,471 \\
\hline Significance level P (Area $=0.5)$ & $<0,0001$ \\
\hline
\end{tabular}

Significance level P (Area $=0.5$

b Binomial exact
Guinea Pig

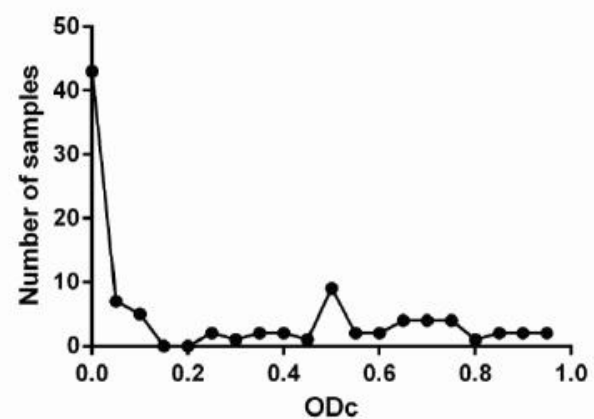

\begin{tabular}{|c|c|c|c|}
\hline ELISA Cut-off & Guinea pig Re & ference popula & \\
\hline DOC 0,25 & + & \begin{tabular}{|l|}
- \\
\end{tabular} & Total \\
\hline+ & 40 & 0 & 40 \\
\hline$\overline{-}$ & 9 & 46 & 55 \\
\hline Total & 49 & 46 & 95 \\
\hline Kappa & Dse $=$ & $\mathrm{DSp}=$ & \\
\hline
\end{tabular}

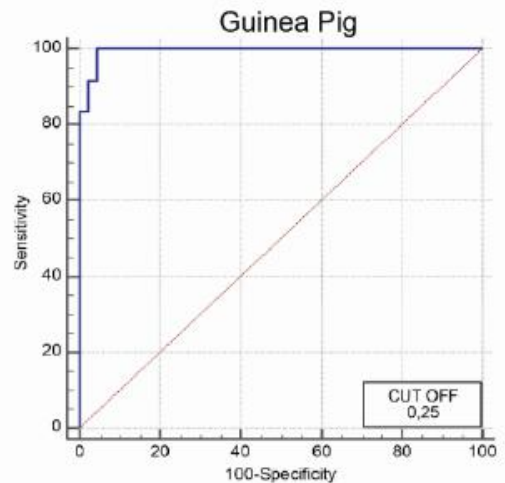

\begin{tabular}{|l|r|}
\hline Area under the ROC curve (AUC) & 0,995 \\
\hline Standard Error a & 0,00435 \\
\hline $95 \%$ Confidence interval b & 0,952 to 1,000 \\
\hline Z statistic & 113,803 \\
\hline Significance level P (Area $=0.5)$ & $<0,0001$ \\
\hline
\end{tabular}

Fig. 1: Cut-offs of the assay for bovine and guinea pig samples and their associated diagnostic sensitivity (DSe) and specificity (DSp). (a) Frequency distribution of the ELISA values obtained, after analyzing the reference populations at a $1 / 40$ dilution. ELISA values were expressed as corrected optical density (ODc). (b) ROC analysis, MedCalc® version 18.11.6 statistical software.

3.2 Feasibility studies, initial repeatability and intermediate precision

The coefficient of variation and the admissible working range of the ODc established for each bovine and guinea pig positive control are detailed in Table 1 . The analysis of variance for the proposed nested model ( 5 assays, 6 plates per assay and 3 replicates per plate)has indicated that the assay had good values of repeatability. The coefficient of variation for bovine and guinea pig positive controls were $15 \%$ and $5.6 \%$, respectively. 
The intermediate precision of the assay given by the overall relative variation for both species was also acceptable (CVbovine $=20.7 \%$ and CVguinea pig $=$ $8.56 \%)$ and met the OIE requirements $(\mathrm{CV}<25 \%)$ (Table1).

\subsection{Detection limit and linearity of the assay}

In the ELISA for bovine and guinea pig samples, the detection limit or absolute analytical sensitivity was estimated by the end-point limiting dilution analysis of positive control sera, assayed in four-fold dilutions on several plates within different independent ELISA runs. The detection limit of the assay was satisfactory and similar for both species (Fig.2a). In both cases the regression analysis from the obtained dose-response curves, has indicated that the assays showed up a range of linear behavior (Fig. 2a), R2 $=0.9$ and R2=0.85 for bovine and guinea pig respectively.
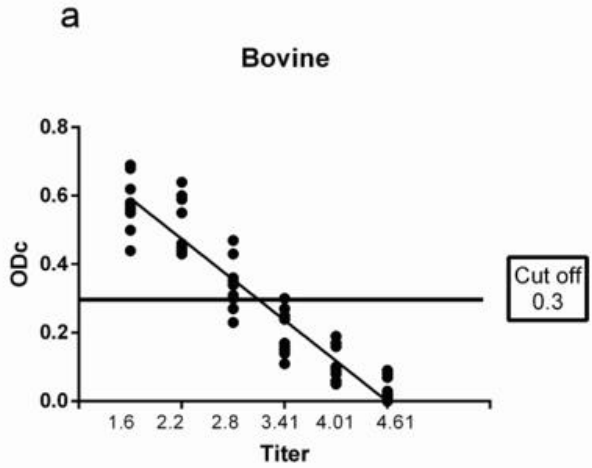

b

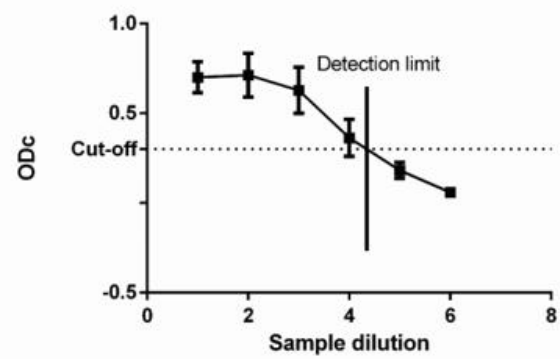

Guinea Pig

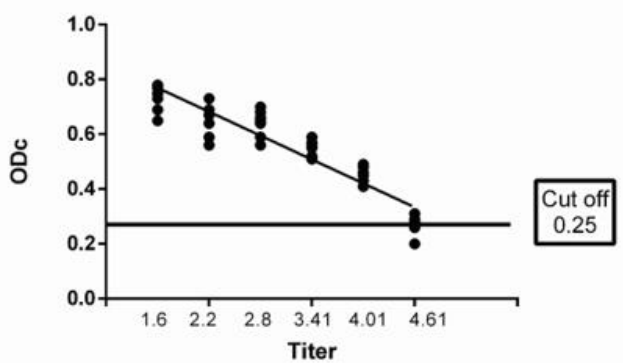

Guinea Pig

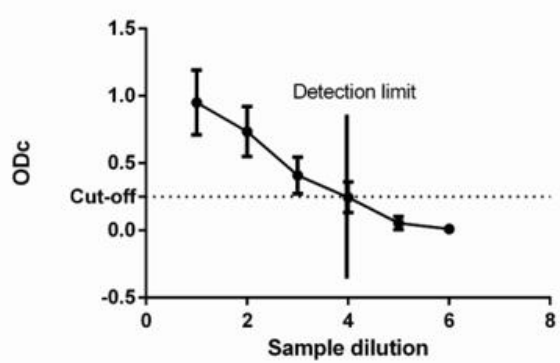

Fig. 2: Dose-response curves. Limit dilution analysis of bovine and guinea pig positive control serum, serial four-fold dilutions starting at 1:40. (a) Each point represents the value obtained for each replicate of the diluted sample in each plate from every independent ELISA run, and the curve is the linear regression; titerswere expressed as the log 10. (b) The response detected by ELISA was expressed as the ODc versus the sample dilution. Each line represents the mean ODc value obtained for the different diluted sample replicates in every independent ELISA run.

3.4 Correlation between the antibody titer obtained by ELISA and HAI assay

There was a significant correlation between the ELISA and the hemagglutination inhibition antibodies titers to BPIV3.
Excluding the negative animals, the Pearson correlation coefficients were lower, but still statistically significant ( $p$ $<0.001)$. The scatterplots, in which both coefficients are reported, are depicted in Fig. 3. 
a

Bovine

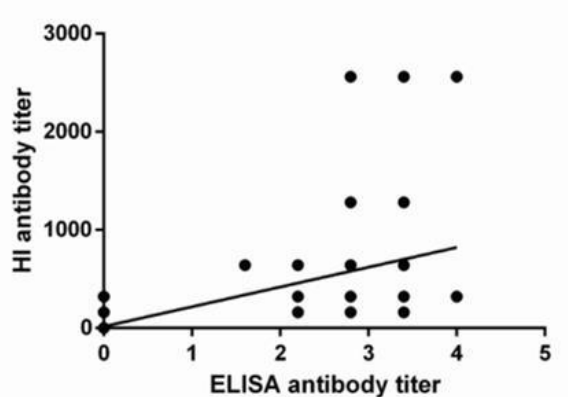

b

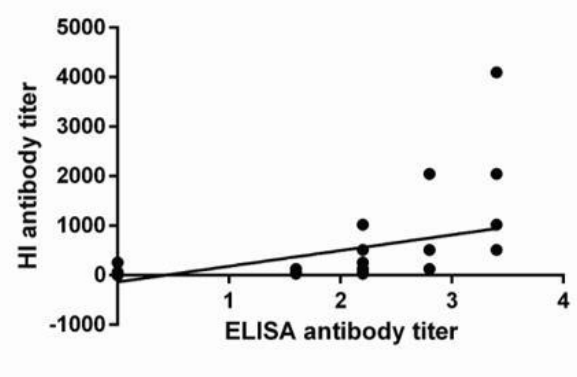

Fig. 3: ELISA relevance defined like the correlation between individual ELISA and neutralizing antibody titers induced by BPIV3 vaccines in groups of 5-10 (a) bovine calves, being sampled 60 days post-vaccination and (b) guinea pigs sampled 30 days post-vaccination. Bovine Pearson correlation coefficient: $0.52(p=1.2 E-6)(n=90)$ and Guinea Pig Pearson correlation coefficient: $0.51(p=9.5 E-4)(n=43)$.

\subsection{Assay reproducibility: inter-laboratory assay}

The inter-laboratory study data have been analyzed with an analysis of variance with 5 ELISA runs, 6 plates and 6 replicates and they were collected in three laboratories (defined in 2.6.6 section). The overall mean for bovine and guinea pig positive controls were 0.733 and 0.674 respectively, both falling within the range of acceptance (Table 1). The reproducibility of the assays expressed as the coefficient of variation was $17 \%$ for bovine and $15 \%$ for guinea pig positive control (Table 1).

\section{DISCUSSION}

In this paper, we successfully validated an indirect ELISA assay by estimating the diagnostic sensitivity and specificity of the assays, the repeatability and precision within a laboratory over time, and also the precision and reproducibility of the assays inter-laboratory. This test will be used to evaluate and quantify antibodies against BPIV3 in bovine sera, thus improving the estimation of prevalence of this virus in herds of our country; and also in guinea pig sera, complementing the HAI tests in the guinea pig model.

Respiratory infections are one of the main causes of disease and death in calves during the first weeks of life and in the first stage of rearing. This is observable in both intensive dairy systems and in farmyard fattening systems, causing significant economic losses. Among the diseases that cause great economic losses of livestock industry there is the fever shipping associated to BPIV3.

Still, little information is available on the epidemiology of BPIV3 in Argentina: some studies demonstrate seropositivity in guanacos ${ }^{17}$. The presence of the three known genotypes of BPIV3 - a, b and c- has been documented in bovine and bubaline cattle in the north of the country, being the first cases in which the sequence is available in our country ${ }^{15}$. For the most part, the diagnosis of BPIV3 is by different serological techniques, including hemagglutination inhibition (HAI): reported seroprevalenceshave been made with this test, which is considered as the gold standard. The HAI principle is based on the ability of the virus, to agglutinate red blood cells, normally from guinea pigs, forming a red button on the cell plates where the assay is performed. However, there are several reports that reveal difficulties in sensitivity, due to low hemagglutination titers in inoculated animals ${ }^{10}$ added to the fact that it requires several steps of molecules inactivation that unspecifically generate hemagglutination, resulting in false positives. The correlation coefficient obtained between both techniques is within acceptable values (Fig. 3).

A faster, cheaper, easier technic to repeat and execute is ELISA. Currently, there are two types of commercially BPI3 ELISA, one is an indirect format for the detection of antibodies in bovinesserum and milk, beeing similar to our assay, that is based on complete viral antigen. The second type is for detecting the virus directly (Biox, Belgium). In addition, the development of an indirect ELISA based on a truncated nucleocapsid protein obtained by recombination has been reported in the literature ${ }^{25}$. On the other hand, a multiplex ELISA was also reported to detect four complex respiratory bovine viruses in bovine serum ${ }^{18}$. In our country these kits are not available and, if they would be wanted to be imported, they would have a very high cost. Nowadays, polyvalent combined inactivated viral vaccines with the virus BPIV3, BoHV1, BDVD and BRSV and bacterial antigens are used as a control strategy for infectious diseases, aimed at facilitating the health 
calendar, and simultaneously, attacking a complex etiological problem, such as bovine respiratory syndrome. However, in many cases, they are not accompanied by objective data that supports their immunogenic quality and efficacy against each of the antigens that compose them. International control agencies (APHIS, USA; EMEACVMP, EU; OIE; VICH) require potency and efficacy tests in the species of application, for the approval of respiratory vaccines; this implies vaccination and infection to susceptible and seronegative cattle. Once the product is approved, the quality control for each serie must be carried out by means of a potency test that defines the immunogenicity of the product in cattle or in another laboratory animal model (in vivo test).

It must be statistically validated, possessing an acceptable degree of concordance with respect to the potency test in the target species, and to be functional as a vaccine efficacy predictive tool. The difficulty to obtain bovines with seronegatives bovine respiratory complex valences, and the high cost of immunogenicity tests in the host species, propose the need to develop a standardized test in laboratory animals. It would allow to compare potency evaluations of each batch of vaccine, in a harmonized way, thus guaranteeing the presence on the market of effective products. To date, the guinea-pig model validated for BPIV3 uses a range of antibody titers established by HAI to categorize the immunogenicity of vaccines ${ }^{4}$. In relation to animal welfare, this test responds to the guidelines of international organizations by replacing and reducing significantly the use of animals in experimental tests ${ }^{11,2,9}$. Not only due to the replacement of bovines by guinea pigs, but also because the change in technique (ELISA for HAI) implies not using red blood cells from animals.

The current situation, about the growth of the national market and the requirement of control organisms and private laboratories, makes it necessary to implement this type of quality control. Currently the technique for quantifying antibodies against BPIV3 validated for the guinea pig model is the HAI. However, due to the variability from each operator reading, the environmental conditions dependence and the fresh red blood cells availability to perform the assay, it was decided to develope these ELISAs. This production will allow us to have the necessary quantity of tests to evaluate the immunogenicity induced by commercial vaccines and to validate the guinea pig model to facilitate the vaccines quality evaluation.

An indirect ELISA for BPIV3 antibody detection in bovine and guinea pigs sera were validated. The statistical validation included the estimation of the assay reliability of the assays using certain cut-off values and their associated diagnostic sensitivity and specificity established in both of the species to make it fit on the assay purpose ${ }^{7}$.

A 0.3 ODc cut-off, $88 \%$ sensitivity and $100 \%$ specificity has been considered suitable to use as a screening method for field surveys of infected animals. The BPIV3 ELISA showed similar performance as the hemagglutination inhibition and it has demonstrated to be more suitable for herd surveys. Similar values were obtained by the indirect biox ELISA; Relative sensitivity: $100 \%$ Relative specificity: $100 \%$. On one hand, the agreement between the two tests is considered excellent (https://www.biox.com/en/bio-k-239-monoscreen-abelisabpi3-indirect-double-wells-p-255/). On the other hand, the NP-N elisa showed a sensitivity of $98.4 \%$ while the specificity was $100 \%{ }^{25}$. None of these carried out reports were as exhaustive statistical studied as this work. In our work a significant correlation was obtained between the ELISA and HAI antibody titers in vaccinated animals analyzed at specific time point after vaccination (60 days for bovines and 30 days for guinea pigs), indicating the relevance of the ELISA's development to be applied on batch to batch vaccine potency testing in both species. To retain international trade markets, vaccination and serology surveys from herds will be mandatory in the near future. This test, the first in ELISA format, will allow us to evaluate the epidemiology of the virus in our region once it is commercially available. The used of this ELISA to detect antibodies in serum, in combination with BPIV3 RT PCR to detect virus genome represent a suitable method of high sensitivity and minimal risk of false negative results 15. As well as this technique constitutes an important monitoring system for the implementation of eradication programs in the region.

In addition, it is well known that the sensitivity and specificity of an assay can varies either with the stage of infection or with immune host status ${ }^{13}$; thus, in positive reference population, we included naturally infected bovines from endemic farms, samples from vaccinated animals and samples from experimentally challenged animals ${ }^{20,23}$.

This study reports the development of an alternative tool to detect antibodies against BPIV3. The additional advantage to the independence of the subjectivity of the operator reading and red blood cells disponibility, is the adjustment of the antibody titers range to assess the quality of vaccines with greater precision. It will be a valuable addition to improve the vaccines available in the market, and consequently, the population immunity to BPIV3 necessary to reduce the high circulation of the virus in the country. 
In the near future this indirect ELISA could be offered as a commercial kit where the plates will be sensitized, dry and stable over time, making available a serological assay for BPIV3 in our region.

\section{CONCLUSIONS}

This assay will be useful to evaluate the seroprevalence in cattle and also for evaluation of the vaccines quality in a guinea pig model. The ELISA showed very good intermediate precision and reproducibility in both species.

\section{ACKNOWLEDGMENTS}

We are very grateful with Natalia Porta and Romina Politzky for the excellent technical assistance.

Funding sources: This work was supported by PID Universidad del Salvador. Evaluación de seroprevalencia a virus parainfluenza 3 en rodeos bovinos de campos de la provincia de Buenos Aires. Validación de ELISA para evaluación de anticuerposbovinos contra PI3. 2016-2019; and for the proyect MAGYP. "Desarrollo de herramientas para la prevención y control de vacunas para Virus de la Diarrea Viral Bovina (VDVB) y Parainfluenza. 20142016.

\section{REFERENCES}

[1] Almeida Vaucher R, Simonetti A, Roehe P. RT-PCR for detection of bovine parainfluenza virus type 3 (bPIV-3). Acta Scientiae Veterinariae. 2008; 36: 215-220.

[2] Akkermans A, Hendriksen C. Statistical evaluation of numbers of animals to be used in vaccine potency testing: a practical approach. Dev Biol Stand. 1999; 101:255-260.

[3] Barbieri S, Rodríguez D, Marin R, Setti W. Serological survey of antibodies against viral diseases of public health interest in llamas (Lama glama) from Jujuy province, Argentina. Rev Argent Microbiol. 2014; 46: 53-57.

[4] Camevet. Guía de prueba de potencia para vacunasbovinasinactivadas que contengan virus parainfluenza 3bovino (pi-3) [Potency Test Guide for Inactivated Bovine Vaccines Containing Bovine Parainfluenza 3 Virus (Pi-3)]. Fundación PROSAIA. 2012

[5] Ellis, J. Bovine parainfluenza- 3 virus. The Veterinary Clinics of North America. Food Animal Practice. 2010; 26(3):575-593

[6] Epstein B. Parainfluenza tipo III enbovinos y ovinos de Argentina. Patología, aislamiento y caracterización [Type III parainfluenza in cattle and sheep from Argentina. Pathology, isolation and characterization]. Rev Argent Microbiol. 1974; 55:397-406.

[7] Greiner M, Gardner I. Application of diagnostic tests in veterinary epidemiologic studies. PrevVet Med. 2000; 45:43-59.
[8] Haanes J, Guimond P, Wardley R. The bovine parainfluenza virus type-3 (BPIV-3) hemagglutinin/ neuraminidase glycoprotein expressed in baculovirus protects calves against experimental BPIV-3 challenge. Vaccine.1997; 15: 730-738.

[9] Halder M, Hendriksen C, Cussler K, Balls M. ECVAM's contributions to the implementation of the Three Rs in the production and quality control of biologicals. Altern Lab Anim. 2002; 30; 93-108.

[10] Haller A, Miller T, Mitiku M, Coelingh K. Expression of the Surface Glycoproteins of Human Parainfluenza Virus Type 3 by Bovine Parainfluenza Virus Type 3, a Novel Attenuated Virus Vaccine Vector. J Virol. 2000; 74:1162611635.

[11] Hendriksen, C. Replacement, reduction and refinement alternatives to animal use in vaccine potency measurement. Expert Rev Vaccines. 2009; 8: 313-322.

[12] Horwood P, Gravel J, Mahony T. Identification of two distinct bovine parainfluenza virus type 3 genotypes. $J$ Gen Virol. 2008; 89: 1643-1648.

[13] Kramps J, Banks M, Beer M, Kerkhofs P, Perrin M, Wellenberg G, Van Oirschot J. Evaluation of tests for antibodies against bovine herpes virus 1 performed in national reference laboratories in Europe .Vet Microbiol. 2004; 102: 169-181.

[14] Lager L, Sadir A, Schudel A. Enfermedadesrespiratoriasvirales de bovinos [Bovine viral respiratory diseases]. $J$ Información $y$ DesarrolloenInvestigaciónAgropecuaria. 1983; 1:55-58.

[15] Maidana S, Lomonaco M, Combessies G, Craig M, Diodati J, Rodriguez D, Parreño V, Zabal O, Konrad J, Crudelli G, Mauroy A, Thiry E, Romera S. Isolation and characterization of bovine parainfluenza virus type 3 from water buffaloes (Bubalusbubalis) in Argentina. BMC Vet Res. 2012; 8; 83.

[16] Marcoppido G, Parreño V, Vilá B. Antibodies to pathogenic livestock viruses in a wild vicuña (Vicugnavicugna) population in the Argentinean Andean altiplano. J Wildl Dis. 2010; 46:608-614.

[17] Marcoppido G, Olivera V, Bok M, Parreño V. Study of the kinetics of antibodies titres against viral pathogens and detection of Rotavirus and Parainfluenza 3 infections in captive crias of guanacos (Lama guanicoe).TransboundEmerg Dis. 2011; 58:37-43.

[18] Mayers J, Sawyer J. Development and evaluation of a multiplex enzyme-linked immunosorbent assay for the detection of antibodies to bovine respiratory diseases on the Meso Scale. Journal of J Vet Diagn Invest. 2012; 24:725729.

[19] Parreño V, López M, Rodriguez D, Vena M, Izuel M, Filippi J, Romera S, Faverin C, Bellinzoni R, Fernandez F, Marangunich L. Development and statistical validation of a guinea pig model for vaccine potency testing against Infectious Bovine Rhinothracheitis (IBR) virus. Vaccine. 2010; 28:2539-2549.

[20] Parreño V, Romera SA, Makek L, Rodriguez D, Malacari D, Maidana S, Compaired D, Combessies G, Vena MM, Garaicoechea L, Wigdorovitz A, Marangunich A, Fernandez 
F. Validation of an indirect ELISA to detect antibodies against BoHV-1 in bovine and guinea-pig serum samples using ISO/IEC 17025 standards. J Virol Methods. 2010; 169:143-153.

[21] Puntel M. Seroprevalencia de infeccionesviralesen llamas (Lama glama) en la república Argentina [Seroprevalence of viral infections in Lama Glama in Argentine]. Rev Argent Microbiol. 1997; 29: 38-46.

[22] Robles C. Relevamientosanitario e implementación de un plan para la prevención y control de enfermedadesenbovinos de productoresruralesminifundistascomunitarios de la provincia de Neuquén, Argentina [Sanitary survey and implementation of a plan for the prevention and control of diseases in cattle from rural small community farmers in the province of Neuquén, Argentina]. Proinder-Inta. 2008.

[23] Romera S, Hilgers L, Puntel M, Zamorano P, Alcon V, Dus Santos M, Blanco Viera J, Borca M, Sadir A. Adjuvant effects of sulfolipocyclodextrin in a squalane-in-water and water-in-mineral oil emulsions for BHV-1 vaccines in cattle. Vaccine. 2000; 19: 132-141.

[24] Snowder G, Van Vleck L, Cundiff L, Bennett G. Bovine respiratory disease in feedlot cattle: environmental, genetic, and economic factors. J Anim Sci. 2006; 84: 1999-2008.

[25] Yang Y, Wang, F, Sun N, Cao L, Zhang S, Zhu W, Wen, Y. Development and evaluation of two truncated recombinant NP antigen based indirect ELISAs for detection of bovine parainfluenza virus type 3 antibodies in cattle. $J$ Virol Methods.2015; 15:47-50. 